SHORT REPORT

\title{
Prospective study of community needlestick injuries
}

N Makwana, F A I Riordan

Arch Dis Child 2005;90:523-524. doi: 10.1136/adc.2004.055624

Fifty three children were referred following community needlestick injuries, August 1995 to September 2003. Twenty five attended for serology six months later. None were positive for HIV, or hepatitis B or C. Routine follow up after community needlestick injury is unnecessary. HIV postexposure prophylaxis should only be considered in high risk children.

C hildren who sustain needlestick injuries outside hospital are seen regularly in accident and emergency (A\&E) departments. ${ }^{12}$ These can be a source of great anxiety for families and schools. Unlike occupational exposure in adult healthcare workers, the infective status of the source case is usually unknown and difficult to establish. There is little information available in the literature on follow up of these children.

The greatest infective risk from a needlestick injury is hepatitis $\mathrm{B}(\mathrm{HBV})$ and hepatitis $\mathrm{C}(\mathrm{HCV}){ }^{3}$ The prevalence of hepatitis in intravenous drug users (IDU) in the UK is $25 \%$ for $\mathrm{HBV}$ and $38 \%$ for $\mathrm{HCV}$, with a risk of transmission of $30 \%$ and $0-7 \%$ respectively following percutaneous injury. ${ }^{4}$ There are marked regional variations in the prevalence of hepatitis C in IDUs from $20 \%$ in the West Midlands to $53 \%$ in London. ${ }^{5}$

The prevalence of human immunodeficiency virus (HIV) infection among IDUs in London during 2001 was one in 2145. Elsewhere in the UK, one in 360-434 IDUs were HIV infected. ${ }^{6}$ An occupational needlestick injury has a risk of transmission of HIV of 3.2/1000 if the source is positive. ${ }^{7}$ It has been estimated that the risk from a community acquired needlestick injury is less than 1:100 000 outside of London. ${ }^{3}$ The Children's HIV Association suggests post-exposure prophylaxis (PEP) for HIV should be considered in some children following needlestick injury, but that the risks of PEP probably outweigh the benefits. ${ }^{3}$

We wished to determine location, mechanism of injury, and age groups affected by childhood out of hospital needlestick injuries and review the outcome of treatment and follow up.

\section{METHODS}

A guideline for managing needlestick injuries in children was introduced into the A\&E departments at Birmingham Heartlands Hospital and Birmingham Children's Hospital in 1997 and 1999 respectively. Birmingham Heartlands Hospital provides secondary care for 100000 children in East Birmingham, while children from all over Birmingham attend A\&E at the Children's Hospital. The guideline was based on the paper of Wyatt and colleagues ${ }^{1}$ and current UK recommendations. ${ }^{8}$ It includes prophylaxis against hepatitis $\mathrm{B}$ and tetanus, referral for counselling, and follow up. All children presenting with needlestick injuries were offered follow up in the paediatric infectious diseases clinic at Birmingham Heartlands Hospital. If, following discussion of risks with parents, repeat testing was required; this was carried out six months from initial exposure. Children with needlestick injuries attending the A\&E department at Birmingham Heartlands Hospital were also referred by a liaison health visitor if this had not already been done. Children referred from other professionals were also seen.

We included all children with needlestick injuries referred to the paediatric infectious diseases clinic at Birmingham Heartlands Hospital between August 1995 and September 2003. Children were identified by a database recording all such referrals. Information was gathered prospectively on patient age, area of Birmingham where injury was sustained, referring party, location of needle, method and anatomical area of injury, management, and follow up data, including the results of serology.

\section{RESULTS}

Fifty three children (63\% male) were referred with a needlestick injury. Median age was 8.4 years (range 1.716.5 years). Of the 53 children, $87 \%$ presented directly to the A\&E departments, while $13 \%$ were referred via the general practitioner (GP) to various medical specialties (paediatrics 3, A\&E 2, genitourinary medicine 2, adult infectious diseases 1). Seventy per cent of injuries occurred between April and September, July being the most common month $(n=11)$. There was an increase in the number of cases presenting each year over the period of the study.

Figure 1 shows the locations where needlestick injuries occurred. The injury at the GP surgery was due to a child putting her hand into a sharps bin. The most common mechanism of injury was playing with a needle found in a public place. Within the home, injury was caused by needles used for measuring blood sugar.

Seventy six per cent of children received the first dose of hepatitis $\mathrm{B}$ vaccine at presentation as per protocol. The maximum time before the first vaccine was given was 14 days in one patient, due to delayed presentation to hospital. Baseline blood sampling was carried out in all patients.

No children received HIV PEP, as there was no requirement in the protocol for this to be discussed or implemented at time of injury.

Forty patients $(75 \%)$ attended a follow up outpatient appointment, and of these $25(63 \%)$ had repeat serology sent at six months. Fourteen (35\%) did not attend the six month appointment and one parent declined six month serology.

Of the 25 patients who had serology sent, all completed three doses of hepatitis B vaccine. None tested positive for HIV, HBV, or HCV.

\section{DISCUSSION}

Needlestick injuries in children outside hospital were first reported in 1987, ${ }^{9}$ with further reports reinforcing the emergence and increase of a new problem. ${ }^{12}$ Our study found that less than $50 \%$ of children referred with a needlestick injury, returned for serology to exclude infection with blood borne viruses six months later. None of those tested had contracted a blood borne infection. This is similar 


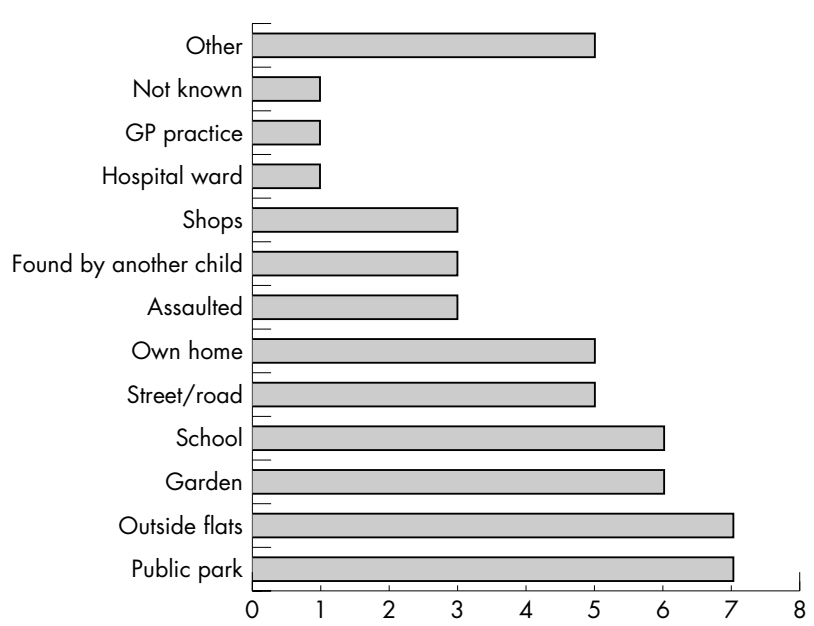

Figure 1 Location where needlestick injury occurred in 53 children referred for follow up. Other includes: car park (1), by a canal (1), on a public bus (1), outside a concert venue (1), friend playing with the needle (1).

to studies from other countries where $41-52 \%$ of children completed serology at six months ${ }^{2}{ }^{10}$ and no children became infected with a blood borne virus after injury.

In our population most children $(76 \%)$ received the first dose of hepatitis $\mathrm{B}$ vaccine immediately following their exposure. The delayed vaccinations appear to be due to a failure to follow the protocol or a delayed presentation to hospital. We cannot be certain about the course completion in children who failed to attend the six month appointment.

HIV PEP remains an unresolved issue. The problem with community needlestick injury is that the infectious status of the source is often unknown. No studies have directly measured the effectiveness of PEP in decreasing the risk of HIV transmission in non-occupational settings, and the potential benefit of PEP in modifying transmission risk is extrapolated from animal and adult occupational exposure studies. Studies examining out of hospital needlestick exposure and HIV seroconversion have shown that no children seroconverted despite not receiving HIV PEP. ${ }^{128}$ Within this population of children were those who sustained injuries from areas with a high prevalence of injecting drug use. We did not identify any cases in our study where HIV PEP would have been advised.

Attention needs to be paid to the prevention of needlestick injuries by publicising the dangers and also urging drug users and patients with illness requiring injections to dispose of their needles responsibly. Many of the reported exposures occurred in public areas, especially parks, streets, and within the vicinity of the home, particularly near flats, similar to Wyatt and colleagues. ${ }^{1}$ There were a number of injuries that occurred in what would be assumed to be safe areas, including the school and within the home. Those injuries sustained at school involved deliberate stabbing by fellow pupils as well as accidents. School education programmes would be important to reduce the incidence of these exposures. Local authorities need to implement educational and community prevention measures designed to reduce the risk of needlestick injuries. Needle exchange programmes have yielded consistently good results in the United States and other countries in reducing the transmission of blood borne pathogens. ${ }^{11}$

The 53 incidents identified in our study are likely to be an underestimate. Not all injuries will have been bought to medical attention and some presenting to the A\&E department at Birmingham Children's Hospital may not have been referred. We cannot comment on whether children who did not return for serology may have been infected. These infections may not present clinically for many years. In view of the low risk and lack of previously documented transmission in this way we assume they are not infected.

\section{Conclusion}

The risk of acquiring HIV from a community needlestick injury is very low in areas outside of inner London. Children with these injuries should be given a course of hepatitis B vaccination. Follow up should only be offered if the parents or child cannot be reassured. HIV PEP should only be considered in those children at very high risk of contracting HIV (that is, injury from a known HIV source with presence of fresh blood on the needle and a deep, penetrating injury). These children should be offered HIV PEP and follow up.

\section{Authors' affiliations}

N Makwana, F A I Riordan, Dept of Child Health, Birmingham

Heartlands Hospital, Birmingham, UK

Competing interests: none declared

Correspondence to: Dr F A I Riordan, Department of Child Health, Birmingham Heartlands Hospital, Bordesley Green East, Birmingham B9 5SS, UK; Andrew.Riordan@heartsol.wmids.nhs.uk

Accepted 17 November 2004

\section{REFERENCES}

1 Wyatt JP, Robertson CE, Scobie WG. Out of hospital needle stick injuries. Arch Dis Child 1994;70:245-6.

2 Nourse CB, Charles CA, McKay M, et al. Childhood needle stick injuries in the Dublin metropolitan area. Ir Med J 1997;90:66-9.

3 Anon. Post exposure prophylaxis (PEP) guidelines for children exposed to blood borne viruses. www. bhiva.org/chiva. Accessed 16 January 2004.

4 Ramsey ME. Guidance on the investigation and management of occupational exposure to hepatitis C. Commun Dis Public Health 1999;2(4):258-62.

5 Health Protection Agency. Shooting up; infections among injecting drug users in the United Kingdom 2003. London: Health Protection Agency, 2004.

6 Unlinked Anonymous Surveys Steering Group. Prevalence of HIV and hepatitis infections in the United Kingdom 2001. London: Department of Health, 2002

7 Tokars Jl, Marcus R, Culver DH. Surveillance of HIV infection and zidovudine use among health care workers after occupational exposure to HIV infected blood. Ann Intern Med 1993;118:913-19.

8 Salisbury D, Begg NT, eds. Immunisation against infectious disease. London: Department of Health, 1996.

9 Walsh SS, Pierce AM, Hart CA. Drug abuse: a new problem. BMJ 1987;295:526-7.

10 Aragon-Pena AJ, Arrazola-Martinez MP, Garcia de Codes A, et al. Prevencion de hepatitis $\mathrm{B}$ y reisgo de infeccion por VIH en ninos accidentados con agujas $y / o$ jeringuillas abandonadas [Hepatitis $B$ prevention and risk of HIV infection in children injured by discarded needles and/or syringes]. Atencion Primaria 1996;17:138-40.

11 Luthrie P, Chen D. A review of programs in North America. In: Stryker J, Smith MD, eds. Dimensions of HIV prevention: needle exchange. Menlo Park, CA: Henry J Kaiser Family Foundation, 1993:11-34. 